\title{
Proposição de um modelo teórico para capacidade de inovação sustentável
}

\section{Proposition of a theoretical model to sustainable innovation capacity}

\author{
Cristiane Froehlich ${ }^{1}$ \\ Claudia Cristina Bitencourt ${ }^{2}$
}

\section{Resumo}

Este estudo trata-se de um ensaio teórico e analisa o desenvolvimento da capacidade de inovação como meio para alavancar a sustentabilidade empresarial. Para atender a esse objetivo, utiliza-se a abordagem das capacidades dinâmicas como auxiliar ao desenvolvimento da capacidade de inovação e mostra como elas podem ser desenvolvidas para suportar a capacidade de inovação. No decorrer da revisão bibliográfica do estudo, verifica-se que essa abordagem esclarece como desenvolver a capacidade de inovação, mas não explica como alavancar a sustentabilidade empresarial. Devido a essa limitação, recorreu-se à abordagem da inovação sustentável, a qual apresenta elementos que ampliam o escopo de atuação da capacidade de inovação para além do pilar econômico, contemplando os pilares ambiental e social da sustentabilidade empresarial. A pesquisa classifica-se em bibliográfica, exploratória e qualitativa. Foram analisados artigos de periódicos que apresentam fator de impacto relevante e livros de autores que são referências nos temas estudados. Utilizou-se, para análise dos textos, a técnica de análise de conteúdo qualitativa, com base nas três etapas básicas indicadas por Bardin (2010): (a) pré-análise; (b) exploração do material; e (c) tratamento dos dados, inferência e interpretação. Este artigo propõe, como principal resultado da pesquisa, a necessidade de desenvolvimento da capacidade de inovação sustentável, integrando as abordagens das capacidades dinâmicas e da inovação sustentável para alavancar a sustentabilidade empresarial.

Palavras-chave: Sustentabilidade empresarial. Capacidade de inovação. Capacidades dinâmicas. Inovação sustentável. Capacidade de inovação sustentável.

Docente e Pesquisadora do Instituto de Ciências Sociais e Aplicadas da Universidade Feevale. Doutora em Administração pela Universidade do Vale do Rio dos Sinos - UNISINOS - Brasil E-mail: froehlich.cristiane@gmail.com

2 Coordenadora do PPGA em Administração da UNISINOS. Doutora em Administração pela UFRGS - Brasil - E-mail: claudiacb@unisinos.br 


\section{Abstract}

The present work analyses the development of innovation capability in a way of leveraging entrepreneurial sustainability. In order to answer study goals, we have used the approach of dynamic capability to help the development of innovation capability and shows how they could be developed to support to innovation capability. Based on the research field, we have verified that this approach clarifies how to develop the innovation capability, but it did not explain how to leverage the entrepreneurial sustainability. Due to this limitation, we have run the approach of sustainable innovation, which presents elements that wide the branches of performance of innovation capabilities beyond the economic pillar, contemplating environmental and social issues of entrepreneurial sustainability. To meet the study objective, the research classifies in bibliographic review, qualitative and exploratory. Were analyzed journals articles which present a relevant impact factor and books of authors that are reference in the topics studied. It was used for the analysis of the texts, the technique of qualitative content analysis, based on three basic steps indicated by Bardin (2010): (a) pre-analysis; (b) exploitation of the material; (c) and data treatment, inference and interpretation. The main research results point out the necessity of developing sustainable innovation capabilities, including the approaches of dynamic capability and also sustainable innovation to leverage the entrepreneurial sustainability.

Keywords: Corporate sustainability. Capacity for innovation. Dynamic capabilities. Sustainable innovation. Sustainable innovation capacity.

\section{Introdução}

Pode-se verificar que há um forte movimento para sustentabilidade no contexto empresarial. Esse movimento é puxado pelo incremento de regulamentações e leis, voltadas principalmente para o meio ambiente, e também para os aspectos sociais. Nesse cenário, algumas organizações identificaram novas oportunidades de negócios, abrindo caminhos para a redução de custos, melhorando os rendimentos e ampliando a participação no mercado (HART, 2005; HART; MILSTEIN, 2004; OPPEN; BRUGMAN, 2011; PRAHALAD, 2005; SIMONS; SLOB; HOLSWILDER; TUKKER, 2001).

Esse movimento ganhou força à medida que novas conferências foram sendo realizadas mundialmente devido às preocupações com 
as questões ambientais e sociais. A primeira que obteve destaque foi a Conferência das Nações Unidas, realizada em 1972, em Estocolmo. Em 1983, foi criada a Comissão Mundial do Meio Ambiente e Desenvolvimento, constituída por empresários e políticos e presidida por GroBrudtland, gerando como resultado o livro Nosso Futuro Comum, de 1987, que introduziu e difundiu o conceito de desenvolvimento sustentável. A partir dessas conferências, novas foram e continuam sendo realizadas em vários países, inclusive no Brasil. Nelas, são estabelecidas metas que são revisadas periodicamente. Desse modo, essas conferências mundiais trouxeram a discussão do desenvolvimento sustentável à tona (CMMAD, 1991; ONU, 2000; PNUD, 2010; UNCED, 1992; UNCED, 2012).

Diante disso, a sustentabilidade pode ser considerada um novo paradigma no contexto empresarial, que objetiva integrar o crescimento econômico, a preservação do meio ambiente e a equidade social como elementos de apoio ao desenvolvimento de longo prazo (BARBIERI; VASCONCELOS; ANDREASSI; VASCONCELOS, 2010). Vale ressaltar que, do mesmo modo que um número crescente de empresas está aderindo a esse paradigma, pode-se dizer que o tema desperta cada vez mais interesse no ambiente acadêmico nacional e internacional, e tornou-se pauta nos governos que participam das comissões globais para discussão das metas, diretrizes e princípios gerais da sustentabilidade, mostrando, assim, a relevância da abordagem na atualidade.

Nesta pesquisa, a sustentabilidade no contexto empresarial é compreendida como um conceito que induz a um modelo de gestão de negócios que considera, no processo de tomada de decisão, além do pilar econômico, os pilares ambiental e social. Desse modo, a organização busca gerar lucro para os acionistas, benefícios ao meio ambiente com a redução dos impactos e a melhoria da qualidade de vida das pessoas com quem mantém interações (SAVITZ; WEBER, 2007).

Conforme Barbieri e Simantob (2007), as primeiras iniciativas empresariais giravam em torno de ações menos complexas, como processos de reciclagem, utilização de produtos reciclados, tratamento de 
efluentes e resíduos. Com o tempo, as discussões sobre sustentabilidade empresarial avançaram e ganharam maior complexidade, envolvendo o desenvolvimento e o ciclo de vida dos produtos e serviços, questões sociais e o interesse dos stakeholders.

Sendo assim, Nidumolu, Prahalad e Rangaswami (2009) e Szekely (2012) reforçam que a ideia da inserção da sustentabilidade nas estratégias organizacionais está ganhando espaço no contexto empresarial. Embora ainda não faça parte da prática corrente, um número crescente de empresas está começando a integrar os pilares da sustentabilidade nas suas estratégias organizacionais a partir do entendimento dos seus impactos na competitividade. Desse modo, empresários e pesquisadores identificaram a necessidade de ampliar os estudos sobre como operacionalizar o conceito de sustentabilidade no ambiente interno da organização.

Para isso, o triple bottom line (pilares econômico, ambiental e social), introduzido por Elkington (1997), tornou-se o conceito central na discussão em torno da sustentabilidade no contexto empresarial. Porém, apenas dizer que os pilares econômico, ambiental e social viabilizam a sustentabilidade não é o suficiente; é preciso encontrar um meio de alavancar a sustentabilidade, através desses três pilares, em práticas associadas às estratégias organizacionais. Com o avanço das pesquisas, vários autores apontaram a importância da inovação como um meio fundamental para atingir esse objetivo (ALMEIDA, 2007; BARBIERI et al., 2010; HART; MILSTEIN, 2004; HALL; VRENDENBURG, 2003; HAUTAMÄKI, 2010; HELLSTRÖM, 2007; NIDUMOLU; PRAHALAD; RANSGASWAMI, 2009; OPPEN; BRUGMAN, 2011; PORTER; KRAMER, 2006; TIDD; BESSANT; PAVITT, 2008).

Dessa forma, a inovação torna-se necessária no contexto da sustentabilidade empresarial, pois pode renovar os produtos e os serviços que a organização oferece ao mercado, e ainda alterar a forma como esses são fabricados ou executados proporcionando benefícios econômicos, ambientais e sociais (TIDD; BESSANT; PAVITT, 2008). Os autores Nidumolu, Prahalad e Ransgaswami (2009, p. 3) complementam 
que a demanda externa por sustentabilidade é uma oportunidade para gerar inovações organizacionais e tecnológicas. Diante disso, "ao tratar da sustentabilidade como objetivo, por meio da inovação, as organizações pioneiras irão desenvolver capacidades que os concorrentes serão pressionados a corresponder" (Referência?).

Esta pesquisa adota como premissa que a inovação é um meio para alavancar a sustentabilidade empresarial, mas, para isso, a inovação necessita ser tratada como uma capacidade organizacional. Em outras palavras, a capacidade de inovação é entendida como uma capacidade organizacional de natureza estratégica.

Dodgson, Gann e Salter (2008) definem a capacidade de inovação como uma habilidade da organização para formulação e planejamento de estratégias de inovação, pois envolve a capacidade de criação, ampliação e modificação de recursos utilizados para inovação. Desse modo, a capacidade de inovação facilita a transformação dos conhecimentos e das aprendizagens em novos produtos, serviços e processos, e introduz no mercado inovações radicais e incrementais com benefícios econômicos, ambientais e sociais.

Tidd, Bessant e Pavitt (2008) sinalizam que, embora a inovação seja cada vez mais percebida como uma forma de construir e sustentar a vantagem competitiva, de maneira isolada, não garante essa vantagem. Sendo assim, a inovação depende da maneira como todo esse processo é conduzido, ou seja, depende dos seus recursos, das rotinas e da capacidade de gerenciamento. Assim, a capacidade de inovação é considerada central para alavancar a sustentabilidade empresarial, ou seja, a capacidade de inovação é importante para as organizações atenderem a necessidade da sustentabilidade demandada pelo ambiente externo.

Oppen e Brugman (2011) reforçam que há necessidade de pesquisas que discutam como as organizações podem inovar de modo a contribuir com a sustentabilidade. As pesquisas que relacionam a intersecção entre as abordagens de inovação e sustentabilidade, no 
sentido de "como" e não apenas apontar a relevância entre elas, ainda necessitam de um maior aprofundamento.

Diante disso, este estudo propõe a seguinte questão: como desenvolver a capacidade de inovação para alavancar a sustentabilidade empresarial? Para responder e compreender a relação entre a capacidade de inovação e a sustentabilidade empresarial, o objetivo geral é analisar o desenvolvimento da capacidade de inovação como meio para alavancar a sustentabilidade empresarial.

Esta pesquisa visa contribuir para avançar essa temática. Para isso, utiliza-se a lente teórica das capacidades dinâmicas, definida como a habilidade de integrar, construir, combinar, proteger e reconfigurar recursos, capacidades e ativos tangíveis e intangíveis da organização em resposta às mudanças ambientais (TEECE, 2007; TEECE; PISANO; SHUEN, 1997).

No decorrer da pesquisa bibliográfica, verificou-se que a capacidade de inovação à luz da lente teórica das capacidades dinâmicas não é suficiente para explicar como alavancar a sustentabilidade empresarial. Assim, foi necessário adicionar uma nova lente teórica ao estudo. Identificou-se a abordagem da inovação sustentável, que apesar de ser recente, apresenta elementos que podem estimular o desenvolvimento da capacidade de inovação não somente com foco econômico, mas contemplando os pilares ambiental e social da sustentabilidade. A inovação sustentável gera renovação ou melhoria de produtos, serviços, processos tecnológicos e organizacionais, promovendo benefícios econômicos, ambientais e sociais (BOS-BROUWERS, 2010). Dessa forma, a inovação sustentável "não se refere apenas à introdução de novidades de qualquer tipo, mas que atendam as múltiplas dimensões da sustentabilidade em bases sistemáticas e que geram resultados positivos para a organização, para a sociedade e para o meio ambiente" (BARBIERI, 2007, p. 105).

A seguir, apresenta-se a fundamentação teórica do estudo, que apresenta a contextualização dos temas sustentabilidade empresarial, capacidade de inovação, capacidades dinâmicas e inovação sustentável. 
Na sequência, apresenta-se o método utilizado. Por fim, os resultados e as contribuições da pesquisa, sugerindo-se um modelo teórico para o desenvolvimento da capacidade de inovação sustentável.

\section{Sustentabilidade empresarial}

A abordagem da sustentabilidade recebeu várias críticas de pesquisadores ao longo da construção de seu percurso. A imprecisão da definição da palavra "sustentável" e os diversos entendimentos da palavra "desenvolvimento", que muitas vezes é confundida com "crescimento econômico", são apontados como críticas.

Pode-se dizer que a definição abrangente de Brundtland sobre desenvolvimento sustentável está na raiz de muitas controvérsias, e há um desacordo entre investigadores de diferentes áreas a respeito de como a sustentabilidade pode ser operacionalizada. Desse modo, a sustentabilidade é um processo contínuo e complexo que necessita de estudos visando explicar como operacionalizar esse conceito.

O conceito de sustentabilidade induz a um novo modelo de gestão de negócios, o qual leva em conta, no processo de tomada de decisão, além do pilar econômico, os pilares social e ambiental. A empresa sustentável é aquela que gera lucro para os acionistas, ao mesmo tempo em que protege o meio ambiente e melhora a vida das pessoas com quem mantém interações (SAVITZ; WEBER, 2007).

No contexto empresarial, destacou-se o tripé da sustentabilidade, ou triple bottom line (PPP - People, Planet, Profit), na expressão original cunhada por Elkington (1997). O uso do conceito de triple bottom line faz com que as empresas busquem não apenas os lucros e os resultados econômicos, mas também o bom desempenho do ponto de vista ambiental e social. Para esse autor, o pilar ambiental da sustentabilidade empresarial se refere à capacidade de uma organização para fazer negócios de uma forma que minimize a poluição e que se reflete na gestão prudente dos recursos naturais. O pilar social engloba uma gama de questões, incluindo as condições de trabalho, a diversidade, a remuneração da força de trabalho, treinamento, entre outras. 
Pode-se afirmar que os principais motivos para o investimento na sustentabilidade no contexto empresarial estão relacionados: à exigência legal e o cumprimento das regulamentações (BARBIERI, 2007; BARBIERI et al.; 2010; HART; MILSTEIN, 2004; OPPEN; BRUGMAN, 2011; PORTER; KRAMER, 2006); à oportunidade de negócio para elevar os rendimentos e a participação no mercado, promovendo o crescimento e a perenidade da organização (HART, 2005; HART; MILSTEIN, 2004; OPPEN; BRUGMAN, 2011; SAVITZ; WEBER, 2007); ao aumento e à proliferação dos stakeholders na sociedade que demandam das organizações as condutas e práticas coerentes com essa nova realidade (HART; MILSTEIN, 2004; PORTER; KRAMER, 2006); aos avanços tecnológicos (HART; MILSTEIN, 2004); à redução dos riscos de prejudicar os clientes, os empregados e as comunidades (SAVITZ; WEBER, 2007); e à melhoria da reputação e do valor da marca no mercado (BARBIERI et al., 2010; HART, 2005; PORTER; KRAMER, 2006; SAVITZ; WEBER, 2007).

Barbieri (2007) diz que o conceito de sustentabilidade tem sido usado por empresas como uma licença para continuar como de costume, fazendo apenas mudanças cosméticas. Destaca-se que existe uma tendência de muitas empresas em considerarem a ecoeficiência e/ou a responsabilidade social como sinônimos de sustentabilidade empresarial. Porém, são critérios que podem ser incorporados no "guarda-chuva" da sustentabilidade, pois muitas organizações estão desenvolvendo práticas de responsabilidade social corporativa para integrar a questão social e complementa que tanto a responsabilidade social corporativa como a ecoeficiência são instrumentos de melhoria contínua (ALMEIDA, 2007).

Porém, o processo de incorporação dessas práticas nas rotinas tem sido lento. Gobble (2012) destaca que a sustentabilidade tornouse uma maneira de fazer negócios, porém, ainda não está claro como operacionalizá-la. Para muitos, a sustentabilidade refere-se apenas aos aspectos ambientais. Além disso, a "sustentabilidade é mais do que um mecanismo de auditoria, é uma excelente fonte de inspiração para inovação e crescimento" (GOBBLE, 2012, p. 65). 
A preocupação com a sustentabilidade está sendo alvo de constantes discussões, e a inovação é apontada como um meio para alavancar a sustentabilidade empresarial, com a ampliação da visão de que apenas analisar o desempenho econômico não é suficiente, devendo ser incluídas na análise as questões ambientais e sociais.

Barbieri e Simantob (2007) reforçam que a inovação é importante para que as organizações possam avançar na questão da sustentabilidade. Assim, entende-se que é necessário desenvolver a capacidade de inovação, pois esta tem enfoque estratégico e possibilita a sistematização de rotinas.

\section{Capacidade de inovação}

Dando continuidade à fundamentação teórica, apresenta-se o objeto do estudo, a capacidade de inovação. A noção de capacidade é importante para aplicar a inovação, pois a capacidade de inovar facilita o desenvolvimento do comportamento das pessoas e da organização para atividades e rotinas sistemáticas de inovação na empresa. A capacidade de inovação é a habilidade de transformar conhecimentos e ideias em novos produtos, processos e sistemas, de forma a beneficiar tanto a empresa como os seus stakeholders (LAWSON; SAMSON, 2001).

Para Dodgson, Gann e Salter (2008), a capacidade de inovação é uma habilidade para formulação e implementação de estratégias de inovação, pois envolve a capacidade de criação, ampliação e modificação de recursos utilizados para inovação, a fim de desenvolver novos produtos, serviços, processos e mercados.

Segundo Tidd, Bessant e Pavitt (2008), para uma capacidade ser considerada estratégica, deve estar a serviço de uma necessidade do usuário para que tenha consumidores, ser única para que os produtos e serviços possam ser cobrados, e ser difícil de reproduzir para que os lucros não sejam consumidos pela concorrência.

A capacidade de inovação facilita a transformação dos conhecimentos e das aprendizagens em novos produtos, serviços e 
processos, e introduz no mercado inovações. Para isso, segundo Tidd, Bessant e Pavitt (2008), a inovação depende da maneira como todo esse processo é conduzido, ou seja, depende dos recursos, das rotinas e da capacidade de gerenciamento.

A capacidade de inovação depende da maneira como todo o seu processo é conduzido. Foram identificados, na bibliografia de inovação, dois modelos, considerados clássicos, que podem ser implementados para conduzir a inovação de modo estruturado, a fim de estabelecer rotinas: a) modelo do funil da inovação (CLARK; WHEELWRIGHT, 1993); e b) modelo de stages-gates (COOPER, 1993). Eles destacam as etapas que podem ser adotadas, desde a captação de ideias até o lançamento da inovação ao mercado, constituindo processos organizacionais para gestão.

Acapacidade de inovação pode serentendida como uma capacidade organizacional de natureza estratégica; em outras palavras, trata-se do alinhamento das práticas de inovação com as estratégias organizacionais. Geralmente, acontece de forma deliberada, sistematizada e utiliza um ou mais modelos para desenvolver a inovação. Desse modo, transforma os conhecimentos e as aprendizagens em novos produtos, serviços e processos, introduzindo no mercado inovações radicais e incrementais.

\section{Capacidades dinâmicas}

Após a apresentação do objeto de estudo, expõe-se a primeira lente teórica utilizada para analisar o desenvolvimento da capacidade de inovação para alavancar a sustentabilidade empresarial. As capacidades dinâmicas tratam da capacidade de identificar e moldar oportunidades e ameaças, aproveitar as oportunidades e manter a competitividade por meio do reforço, combinação, proteção e, quando necessário, da reconfiguração dos ativos tangíveis e intangíveis da organização (TEECE, 2007).

Vale ressaltar que essa abordagem é indicada para o estudo de estratégias organizacionais, sendo associada ao contexto de mudança e 
inovação, e caracteriza-se pela mobilização de recursos e capacidades para atender às estratégias de negócios, conforme o dinamismo do ambiente. Devido a essas características, a abordagem é condizente com a natureza da capacidade de inovação para gerar oportunidades de renovação de negócios.

Lawson e Samson (2001) complementam que as organizações que possuem capacidades dinâmicas influenciam e moldam o mercado por meio da capacidade de inovação e possuem facilidade para se adaptar e atender às demandas do mercado.

Então, a fim de compreender como desenvolver a capacidade de inovação para alavancar a sustentabilidade empresarial, necessita-se identificar quais capacidades dinâmicas são necessárias para auxiliar o desenvolvimento da capacidade de inovação. Desse modo, entende-se que a lente teórica das capacidades dinâmicas é um eixo fundamental para o desenvolvimento da capacidade de inovação, consolidando-a como uma capacidade organizacional que alavanca a sustentabilidade empresarial.

Vale destacar que a abordagem das capacidades dinâmicas recebe críticas, pois a conceituação é vaga e tautológica. A tautologia pode ser causada pelo fato de o conceito ser mais teórico e de difícil operacionalização. A ambiguidade é apresentada em várias definições das capacidades dinâmicas, com alto nível de abstração. Também há confusões entre os conceitos, devido aos diferentes termos associados, tais como capacidades, habilidades, competências etc. As dificuldades vão desde encontrar uma definição comum aceita até definir a função das capacidades dinâmicas.

Conforme Helfat e Peteraf (2011), a investigação da abordagem das capacidades dinâmicas é ampla e complexa porque abrange domínios sobre o processo da estratégia organizacional e abrange vários níveis de análise, como o de decisão, processos, rotinas organizacionais, mudanças ambientais e competitividade. Helfat e Peteraf (2011, p. 92) reforçam que "as deficiências revelam o estágio inicial de desenvolvimento de uma teoria e que há muito trabalho a 
ser feito, pois as teorias que apresentam complexidades não vêm préembaladas, e muitas ainda se desenvolvem lentamente". Apesar das críticas em relação à abordagem das capacidades dinâmicas, segundo Ambrosini, Bowman e Collier (2009), a importância teórica e prática de desenvolvimento e aplicação das capacidades dinâmicas para sustentar a vantagem competitiva de uma empresa em ambientes complexos e voláteis levou essa questão para o primeiro plano das agendas de pesquisa de muitos investigadores, mostrando a sua relevância na atualidade.

Corroborando com Ambrosini, Bowman e Collier (2009), pode-se dizer que a abordagem das capacidades dinâmicas é capaz de explicar como determinadas organizações conseguem identificar oportunidades no ambiente externo e incorporá-las em suas rotinas e processos, por meio do gerenciamento e da mobilização de recursos, obtendo resultados positivos, enquanto outras não desenvolvem essa capacidade. Além disso, a teoria das capacidades dinâmicas auxilia e oferece suporte para a compreensão do desenvolvimento da capacidade de inovação.

Nesse sentido, Teece (2007) desenvolveu uma classificação que visa à integração das estratégias organizacionais com a inovação, contribuindo para a capacidade de inovação se consolidar como uma capacidade organizacional de natureza estratégica.

Teece (2007) publicou em 2007 o artigo "Explicating dynamic capabilities: the nature and microfoundations of enterprise performance". Neste artigo, o autor apresentou um framework com o objetivo de integrar as abordagens de estratégia e de inovação, para fornecer "um modelo que destaca a gestão de recursos críticos para sustentar a aptidão evolutiva da empresa" (2007, p. 1322). O framework de Teece (2007) apresenta três capacidades dinâmicas, e cada uma delas possui microfundamentos que facilitam sua operacionalização. O Quadro 1 apresenta essas capacidades. 
Quadro 1 - As capacidades dinâmicas e os seus microfundamentos

\begin{tabular}{|c|c|}
\hline Capacidades Dinâmicas & Microfundamentos \\
\hline $\begin{array}{l}\text { Capacidade de identificar o } \\
\text { contexto do ambiente (sensing) }\end{array}$ & $\begin{array}{l}\text { - Processos para dirigir trabalhos internos de } \\
\text { pesquisa e desenvolvimento. } \\
\text { - Processos para parcerias com fornecedores } \\
\text { para complementar as inovações da } \\
\text { organização. } \\
\text { - Processos para explorar desenvolvimentos } \\
\text { científicos e tecnológicos exógenos. } \\
\text { - Processos para identificar segmentos de } \\
\text { mercado alvo, necessidade dos clientes e } \\
\text { geração de inovações que sejam de interesse } \\
\text { dos clientes. }\end{array}$ \\
\hline $\begin{array}{l}\text { Capacidade de aproveitar/ } \\
\text { incorporar as oportunidades } \\
\text { (seizing) }\end{array}$ & $\begin{array}{l}\text { - Soluçães para os clientes e o modelo de } \\
\text { negócio. } \\
\text { - Seleção das fronteiras organizacionais. } \\
\text { - Rotinas para seleção de protocolos de tomada } \\
\text { de decisão. } \\
\text { - Rotinas para construir lealdade e } \\
\text { comprometimento. }\end{array}$ \\
\hline $\begin{array}{l}\text { Capacidade de gerenciar } \\
\text { ameaças e transformações } \\
\text { (reconfiguring) }\end{array}$ & $\begin{array}{l}\text { - Descentralização e decomposição. } \\
\text { - Coespecialização. } \\
\text { - Governança e o gerenciamento do } \\
\text { conhecimento. }\end{array}$ \\
\hline
\end{tabular}

Fonte: Adaptado de Teece (2007).

Diante disso, o estudo propõe o primeiro pressuposto da pesquisa: (P1): a identificação das capacidades dinâmicas propostas por Teece (2007) explica a capacidade de inovação de maneira sustentável.

Teece, Pisano e Shuen (1997) apresentaram três elementos que contribuem para o desenvolvimento das capacidades dinâmicas e, paralelamente, suportam a capacidade de inovação: (a) trajetória, ou seja, o caminho percorrido e o seu repertório de rotinas construído ao longo dos anos; (b) posição, que se refere ao posicionamento estratégico da empresa; e (c) processos gerenciais e organizacionais, que possuem três funções, coordenação e integração das atividades, aprendizagem, reconfiguração e transformação. 
Conforme Teece, Pisano e Shuen (1997), as capacidades são intrínsecas aos processos organizacionais, mas a possibilidade de criação de vantagem competitiva com base nesses processos, em um dado momento, depende da posição da empresa em termos de seus ativos e de sua trajetória evolutiva.

\section{Inovação sustentável}

A abordagem da inovação sustentável está em sua fase inicial de desenvolvimento, portanto, não há ainda um escopo amplo de publicações. A maioria das publicações existentes trata sobre a ecoinovação, mas já é possível identificar algumas críticas na revisão bibliográfica. Com relação à conceituação, a definição de inovação sustentável apresenta uma demarcação conceitual considerada fraca e é considerada uma abordagem complexa, que apresenta teorias insuficientes em relação à sua operacionalização (BARBIERI et al., 2010; HANSEN; GROSSEDUNKER; REICHWALD, 2009; SZEKELY, 2012).

A abordagem da inovação sustentável é recente. Essa denominação começou a ser empregada timidamente nas pesquisas acadêmicas no início dos anos 2000. Pode-se afirmar que a inovação sustentável origina-se da abordagem da ecoinovação, introduzida em 1996 pelos autores Claude Fussler e Peter James, que apresentaram o conceito de ecoinovação a partir da perspectiva da sustentabilidade, entretanto, com foco nos pilares econômico e ambiental (FLUSSLER; JAMES, 1996).

Esta pesquisa adota o termo "inovação sustentável" e corrobora com o conceito proposto por Bos-Brouwers (2010). Desse modo, a inovação sustentável é entendida como uma inovação que gera renovação ou melhoria de produtos, serviços, processos tecnológicos ou organizacionais, estruturas e modelos de negócios em curto e longo prazo, melhorando o desempenho econômico, ambiental e social. Sendo assim, a integração dos três pilares nas estratégias e nas ações das organizações distingue as inovações sustentáveis das inovações convencionais e amplia essa abordagem. 
Porém, Barbieri et al. (2010) ressaltam que inovar seguindo as três dimensões da sustentabilidade ainda não é a regra, até porque a inclusão dos pilares ambiental e social requer novos instrumentos e modelos de gestão, que só recentemente começaram a ser desenvolvidos, mas não estão consolidados.

Diante do exposto, forma-se o segundo pressuposto (P2): a inovação deve contemplar necessariamente os três pilares do triple bottom line (econômico, ambiental e social) para ser considerada uma inovação sustentável.

Hansen, Grosse-Dunker e Reichwald (2009) criaram o modelo do cubo da inovação sustentável, o qual trata de um framework para a inovação que mostra os efeitos da sustentabilidade em diferentes áreas da organização. O modelo apresenta alguns elementos que os autores consideram importantes para inovação sustentável, destacados no Quadro 2.

Quadro 2 - Elementos da inovação sustentável

\begin{tabular}{|l|l|}
\hline Elementos & \multicolumn{1}{|c|}{ Descrição } \\
\hline $\begin{array}{l}\text { Integração dos critérios } \\
\text { de sustentabilidade }\end{array}$ & $\begin{array}{l}\text { Refere-se à necessidade de considerar os impactos } \\
\text { econômicos, ambientais e sociais no desenvolvimento } \\
\text { de inovações para o mercado. Sugere-se equilibrar as } \\
\text { três dimensões, prestando maior atenção aos pilares } \\
\text { ambiental e social. }\end{array}$ \\
\hline \multirow{3}{*}{$\begin{array}{l}\text { Integração dos } \\
\text { stakeholders }\end{array}$} & $\begin{array}{l}\text { A empresa deve fomentar a participação dos } \\
\text { stakeholders nos processos de criação de inovações, } \\
\text { pois os envolvidos no processo podem avaliar os } \\
\text { possíveis impactos sobre a sociedade ou o meio } \\
\text { ambiente antes mesmo que as novidades sejam } \\
\text { lançadas no mercado, e essa integração pode } \\
\text { favorecer o compartilhamento de conhecimentos e } \\
\text { inspirar inovações. }\end{array}$ \\
\hline $\begin{array}{l}\text { Incorporação de } \\
\text { ferramentas e processos }\end{array}$ & $\begin{array}{l}\text { Permite a implantação da inovação sustentável } \\
\text { e desenvolvem novos mecanismos de acesso ao } \\
\text { conhecimento, a especialização, a aquisição de novos } \\
\text { recursos e as capacidades para responder às novas } \\
\text { exigências de conhecimento. }\end{array}$ \\
\hline
\end{tabular}




\begin{tabular}{|l|l|}
\hline $\begin{array}{l}\text { Investigação do ciclo dos } \\
\text { produtos }\end{array}$ & $\begin{array}{l}\text { Verifica-se a origem da matéria-prima, as } \\
\text { consequências ambientais e sociais de produção e } \\
\text { consumo e o destino dos produtos no final de sua vida } \\
\text { útil. }\end{array}$ \\
\hline $\begin{array}{l}\text { Incremento do sistema } \\
\text { de produto e serviço }\end{array}$ & $\begin{array}{l}\text { A empresa deve pensar no desenvolvimento de } \\
\text { inovações que deslocam o foco empresarial de vender } \\
\text { apenas produtos físicos, de modo que os consumidores } \\
\text { comprem a utilidade ou serviço oferecido pelo produto. } \\
\text { As empresas podem agregar uma maior quantidade } \\
\text { produzir de forma customizada, evitar desperdícios de } \\
\text { recursos, e construir relações de longo prazo com os } \\
\text { clientes. }\end{array}$ \\
\hline $\begin{array}{l}\text { Marketing direcionado à } \\
\text { inovação sustentável }\end{array}$ & $\begin{array}{l}\text { Refere-se ao desafio de criar e projetar novas } \\
\text { necessidades sustentáveis e mudar o estilo de vida } \\
\text { atual. }\end{array}$ \\
\hline $\begin{array}{l}\text { Sensibilização } \\
\text { no contexto da }\end{array}$ & $\begin{array}{l}\text { Significa integrar a sustentabilidade nos processos } \\
\text { organizacionais e nos comportamentos por meio das } \\
\text { lideranças para formação de uma cultura favorável } \\
\text { à mudança. Ou seja, sensibilizar os tomadores de } \\
\text { decisão envolvidos nos processos de inovação } \\
\text { para as questões da sustentabilidade para que eles } \\
\text { possam identificar oportunidades demandadas por } \\
\text { stakeholders e assim diminuir os riscos das inovações. }\end{array}$ \\
\hline
\end{tabular}

Fonte: Adaptado de Hansen, Grosse-Dunker e Reichwald (2009).

A partir da lente teórica da inovação sustentável, esta pesquisa adotou os elementos propostos por Hansen, Grosse-Dunker e Reichwald (2009), que não apresentam as etapas de como desenvolver a inovação sustentável, mas mostram os elementos que devem ser considerados nessas etapas e que podem dar um caráter de sustentabilidade à capacidade de inovação para alavancar a sustentabilidade empresarial.

Diante disso, pode-se dizer que as empresas com capacidade de inovação consolidada podem se posicionar para se tornar organizações orientadas para a inovação sustentável e, assim, alavancar a sustentabilidade empresarial. Desse modo, a capacidade de inovação desenvolvida é um antecedente importante para a inovação sustentável, que contribui, a partir da ampliação da perspectiva da capacidade de 
inovação com enfoque somente na sustentabilidade econômica, para o enfoque nos três pilares da sustentabilidade empresarial.

Para Bos-Brouwers (2010), a inovação sustentável pode ser considerada como o conceito que contribui para a promoção da sustentabilidade empresarial, pois nesse conceito, as empresas integram as questões econômica, ambiental e social em suas operações de negócios, a fim de criar valor, garantir a longevidade e incorporar as preocupações dos stakeholders por meio do lançamento de produtos e serviços, além de melhorias nos processos, na estrutura e no modelo de gestão. Nesse sentido, o terceiro pressuposto da pesquisa (P3) é: a inovação sustentável contribui para a promoção da sustentabilidade empresarial.

\section{Procedimentos metodológicos}

Esta pesquisa classifica-se em bibliográfica, exploratória e qualitativa. Para a realização deste ensaio teórico, fez-se a revisão da literatura, foram analisados artigos de periódicos que apresentam fator de impacto relevante e livros de autores que são referência nos temas estudados, com o intuito de explorar os materiais já publicados.

Para análise do material bibliográfico, utilizou-se a técnica de análise de conteúdo qualitativa que compreende três etapas, conforme indicadas por Bardin (2010): (a) pré-análise; (b) exploração do material; e (c) tratamento dos dados, inferência e interpretação. Conforme Flick (2004, p. 201), esse é um dos procedimentos clássicos para analisar o material textual: "Um de seus aspectos essenciais é o emprego de categorias, obtidas com frequência, de modelos teóricos."

$\mathrm{Na}$ primeira etapa, selecionaram-se artigos em Journals internacionais e brasileiros disponibilizados na base EBSCO, em biblioteca digital na web, que apresentavam no título, no resumo e nas palavraschave as expressões "sustentabilidade empresarial", "capacidade de inovação", "capacidades dinâmicas" e "inovação sustentável”. Ainda 
foram identificados livros de autores que são referências nos temas estudados.

Na segunda etapa, procedeu-se à leitura integral dos artigos préselecionados na fase inicial, com o intuito de identificar os artigos e livros que contribuem diretamente para aprofundar a pesquisa sobre o objeto de estudo. Na terceira e última etapa, os textos selecionados foram lidos novamente e submetidos aos critérios de análise de conteúdo qualitativa. O período de coleta e de análise dos dados foi realizado entre 2013 e 2014.

Para facilitar a análise de conteúdo dos dados, foram definidas, com base no referencial teórico, quatro categorias: sustentabilidade empresarial, capacidade de inovação, capacidades dinâmicas e inovação sustentável. A categorização é apontada por Selltiz, Jahoda e Cook (1965) como uma técnica eficaz para a organização e redução dos dados, por meio do agrupamento das informações e de um número limitado de categorias.

Vale ressaltar que a pesquisa qualitativa envolve a interpretação de um fenômeno organizacional que não pode ser rigorosamente medido em termos de quantidade, frequência ou intensidade (FLICK, 2004). Para Shah e Corley (2006), a abordagem qualitativa é considerada importante para construir novas teorias ou refinar as teorias existentes, além de ser um conjunto de coleta de dados e técnicas de análise que pode ser usado para fornecer descrição, construir teoria e testar teoria. Desse modo, a pesquisa qualitativa permite que o investigador descubra novas relações, revele e compreenda processos complexos, e ilustre a influência do contexto social.

\section{Resultados, conclusões e contribuições da pesquisa}

Esta pesquisa adotou como premissa que a inovação pode ser o meio para alavancar a sustentabilidade empresarial, mas, para isso, a inovação necessita ser tratada como uma capacidade organizacional. 
Em outras palavras, a capacidade de inovação deve ser entendida como uma capacidade organizacional de natureza estratégica.

Assim sendo, a capacidade de inovação é considerada central para alavancar a sustentabilidade empresarial, ou seja, a capacidade de inovação é importante para as organizações atenderem à necessidade da sustentabilidade demandada pelo ambiente externo.

$\mathrm{Na}$ revisão bibliográfica, foram identificadas três capacidades dinâmicas que auxiliam o desenvolvimento da capacidade de inovação, propostas por Teece (2007), denominadas de: a) capacidade de identificar o contexto do ambiente (sensing); b) capacidade de aproveitar/incorporar oportunidades (seizing); e c) capacidade de gerenciar ameaças e transformações (reconfiguring). Pode-se dizer que essas capacidades dinâmicas formadas por microfundamentos auxiliam o desenvolvimento da capacidade de inovação. Teece (2007) elencou essas capacidades após estudos realizados visando à integração da inovação com a estratégia organizacional. Esta pesquisa verificou que a lente teórica das capacidades dinâmicas é um eixo fundamental para o desenvolvimento da capacidade de inovação, consolidando-a como uma capacidade organizacional.

Essa lente teórica é indicada para o estudo de estratégias organizacionais, é associada ao contexto de mudança e de inovação, e caracteriza-se pela mobilização de recursos e capacidades continuamente para atender às estratégias de negócios conforme o dinamismo do ambiente. Devido a essas características, a abordagem das capacidades dinâmicas é condizente com a natureza da capacidade de inovação para gerar oportunidades de renovação de negócios.

Para analisar como são desenvolvidas essas capacidades dinâmicas, foram identificados na abordagem teórica os elementos chamados de trajetória, posição, processos gerenciais e organizacionais, propostos por Teece, Pisano e Shuen (1997). A análise desses elementos permite verificar se há sistematização da inovação, e se é um fator estratégico para organização capaz de mobilizar recursos 
constantemente, ou seja, mostra o processo dinâmico da capacidade de inovação.

Vale ressaltar que, durante a revisão bibliográfica das lentes teóricas estudadas, foram estabelecidos três pressupostos de pesquisa para nortear o desenvolvimento do estudo.

O pressuposto 1 diz que a identificação das capacidades dinâmicas propostas por Teece (2007) explica a capacidade de inovação de maneira sustentável.

Esse pressuposto não foi confirmado. As três capacidades dinâmicas propostas por Teece (2007), chamadas de capacidade de identificar o contexto do ambiente (sensing), capacidade de aproveitar/ incorporar as oportunidades (seizing), e capacidade de gerenciar ameaças e transformações (reconfiguring), formadas por microfundamentos, auxiliam o desenvolvimento da capacidade de inovação.

Pode-se dizer que a abordagem das capacidades dinâmicas é reconhecida como uma lente teórica estratégica que explica a natureza da capacidade de inovação, ou seja, se essa capacidade é operacional ou estratégica. Ainda explica se há alinhamento entre as estratégias organizacionais e as inovações. Enfim, as capacidades dinâmicas auxiliam o desenvolvimento da capacidade de inovação organizacional de natureza estratégica.

Entretanto, essas capacidades dinâmicas não explicam de que forma a sustentabilidade pode ser alavancada por meio da capacidade de inovação. Isso porque a abordagem das capacidades dinâmicas limitase à questão da mobilização de recursos e capacidades para promoção contínua da vantagem competitiva e do crescimento sustentável da organização com foco no pilar econômico, não contemplando os pilares ambiental e social da sustentabilidade, nos conceitos e nas classificações.

A partir da análise do desenvolvimento da capacidade de inovação à luz da abordagem das capacidades dinâmicas, verificou-se que essa lente teórica apresenta as capacidades dinâmicas necessárias para o desenvolvimento da capacidade de inovação e os elementos para o 
desenvolvimento dessas capacidades para suportar a capacidade de inovação. Entretanto, não foi possível analisar como a capacidade de inovação alavanca a sustentabilidade empresarial.

Sendo assim, encontra-se dificuldade em relacionar a capacidade de inovação com a sustentabilidade empresarial, utilizando somente a lente teórica das capacidades dinâmicas. Com isso, recorreu-se à lente teórica da inovação sustentável para a busca de elementos que possam contribuir e ampliar a perspectiva da capacidade de inovação posicionando-a para a sustentabilidade. Essa abordagem é relativamente nova e fundamentada na lente da ecoinovação. A partir do estudo da inovação sustentável, pode-se verificar como a capacidade de inovação se relaciona com a sustentabilidade empresarial.

A partir da revisão bibliográfica da abordagem da inovação sustentável, formou-se o segundo pressuposto da pesquisa, (P2): a inovação deve contemplar necessariamente os três pilares do triple bottom line (econômico, ambiental e social) para ser considerada uma inovação sustentável.

O segundo pressuposto foi confirmado. Os autores dessa abordagem enfatizam que a inovação deve contemplar os três pilares para ser considerada uma inovação sustentável. Bos-Brouwers (2010) define a inovação sustentável como uma inovação que não só proporciona um desempenho econômico melhor, mas também um desempenho ambiental e social, em curto e longo prazo.

Mas vale ressaltar que a questão a discutir não se trata de a inovação contemplar igualmente os três pilares. A reflexão deve partir da consideração e das preocupações desses aspectos da estratégia de concepção do produto, serviço, processo etc. alinhada à sua capacidade de inovação.

O último pressuposto da pesquisa trata (P3) é: a inovação sustentável contribui para promoção da sustentabilidade empresarial.

Pode-se dizer que sim, pois a lente teórica da inovação sustentável apresenta elementos que podem ampliar a perspectiva da capacidade 
de inovação para atender aos princípios da sustentabilidade. Em outras palavras, pode-se afirmar que a inovação sustentável pode fomentar a capacidade de inovação, dando um caráter de sustentabilidade para essa capacidade a partir da análise dos seguintes elementos: pilares da sustentabilidade, parcerias, ferramentas e processos, ciclo dos produtos e serviços, e sensibilização para uma sustentabilidade/cultura visando à inovação sustentável.

É importante ressaltar que a abordagem da inovação sustentável apresenta limitações em relação a como desenvolver a capacidade de inovação por meio da gestão e de processos, e estes são apresentados pela lente das capacidades dinâmicas.

Portanto, a partir da análise realizada, apresenta-se na Figura 1 o modelo teórico proposto da pesquisa.

Figura 1 - Modelo teórico da pesquisa

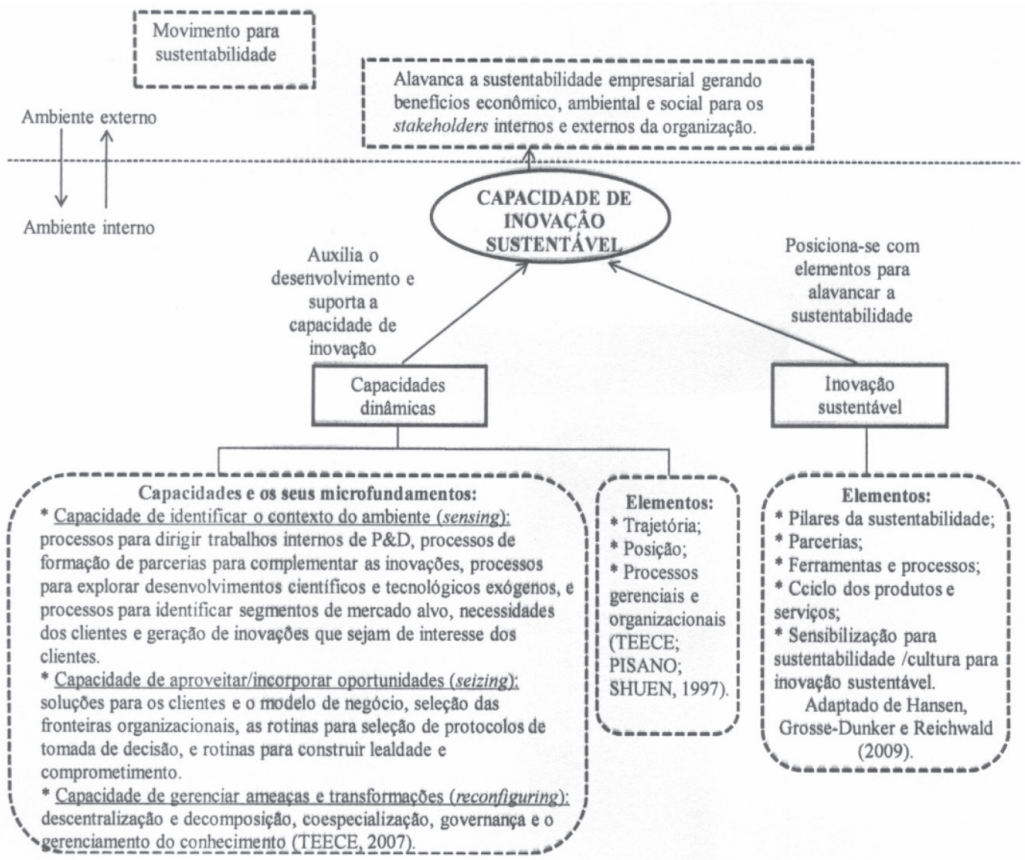

Fonte: Elaborado pelas autoras. 
A Figura 1 apresenta o modelo teórico desta pesquisa e propõe a Capacidade de Inovação Sustentável como resultado da pesquisa.

A Capacidade de Inovação Sustentável acontece a partir da análise da abordagem das capacidades dinâmicas, que apresenta as capacidades dinâmicas necessárias e como desenvolvê-las para suportar a capacidade de inovação. Vale ressaltar que a abordagem das capacidades dinâmicas não dá o caráter da sustentabilidade para a capacidade de inovação, ou seja, não explica a capacidade de inovação no contexto da sustentabilidade, devido à sua origem ter enfoque no fator econômico. Essa lente analisa a questão processual e de gestão da mobilização de recursos em resposta às mudanças ambientais.

Com isso, foi necessário incluir a lente teórica da inovação sustentável, a qual apresenta elementos que ampliam a perspectiva da capacidade de inovação, contemplando os três pilares da sustentabilidade. Para analisar como a inovação sustentável se desenvolve, foi preciso recorrer à abordagem da inovação, pois a abordagem da inovação sustentável não apresenta modelos que mostram como desenvolvê-la.

Sendo assim, esta pesquisa sugere que, para desenvolver a inovação sustentável, é preciso utilizar modelos de inovação constituídos por etapas e processos nas organizações, a fim de constituir rotinas e práticas estratégicas para a inovação sustentável se posicionar como uma lente teórica que contribui para sustentabilidade empresarial.

Em síntese, a Capacidade de Inovação Sustentável necessita ser suportada pelas duas lentes teóricas (capacidades dinâmicas e inovação sustentável), que se complementam para alavancar a sustentabilidade empresarial. Sendo assim, pode ser definida como a capacidade de identificar, desenvolver e gerenciar inovações contemplando os pilares do triple bottom line (econômico, ambiental e social) para alavancar a sustentabilidade empresarial, gerando benefícios para os stakeholders no escopo de atuação da organização.

Mais pontualmente, pode-se destacar como contribuição teórica do estudo: 
- Apresentação do modelo teórico de pesquisa que propõe a Capacidade de Inovação Sustentável (Figura 1).

- Formulação do conceito de Capacidade de Inovação Sustentável: que pode ser definida como a capacidade de identificar, desenvolver e gerenciar inovações contemplando os pilares do triple bottom line (econômico, ambiental e social) para alavancar a sustentabilidade empresarial gerando benefícios para os stakeholders no escopo de atuação da organização.

- A adaptação dos elementos sugeridos por Hansen, GrosseDunker e Reichwald (2009) também pode ser considerada uma contribuição teórica. Os elementos adaptados e sugeridos são: pilares da sustentabilidade, parcerias, ferramentas e processos, ciclo dos produtos e serviços, sensibilização para sustentabilidade/cultura para inovação sustentável.

Finalmente, o estudo apresenta como limitação o fato de constituir-se uma revisão teórica. Como sugestão de estudos futuros, recomenda-se analisar a Capacidade de Inovação Sustentável em organizações industriais e de serviços para verificar como se comporta essa capacidade proposta pelo estudo.

\section{Referências}

ALMEIDA, F. Os desafios da sustentabilidade. Rio de Janeiro: Elsevier, 2007.

AMBROSINI, V.; BOWMAN, C.; COLLIER, N. Dynamic Capabilities: an exploration of how firms renew their resource base. British Journal of Management, England, v. 20, n.1, p. 9-24, Marc. 2009.

BARBIERI, J. C.; SIMANTOB, M. Organizações inovadoras sustentáveis: uma reflexão sobre o futuro das organizações. São Paulo: Atlas, 2007. 
BARBIERI, J. C. Organizações inovadoras sustentáveis. In: BARBIERI, J. C.; SIMANTOB, M. Organizações inovadoras sustentáveis: uma reflexão sobre o futuro das organizações. São Paulo: Atlas, 2007.

BARBIERI, J. C.; VASCONCELOS, I. F. G. de; ANDREASSI, T.; VASCONCELOS, F. C. de. Inovação e sustentabilidade: novos modelos e proposições. RAE - Revista Administração Eletrônica, São Paulo, v. 50, n. 2, p. 146-154, abr./jun. 2010.

BARDIN, L. Análise de conteúdo. Lisboa: Edições 70, 2010.

BOS-BROUWERS, H. Sustainable innovation processes within small and medium-size enterprises. Amsterdam: Vrije Universiteit, 2010.

CLARK, K. B.; WHEELWRIGHT, S. C. Managing new product and process development: text and cases. New York: Free Press, 1993.

COMISSÃO MUNDIAL SOBRE O MEIO AMBIENTE E DESENVOLVIMENTO. Nosso Futuro Comum. 2 ed. Rio de Janeiro: Fundação Getúlio Vargas, 1991.

COOPER, R. G. Winning at new products: accelerating the process from idea to launch. 2 edition. Boston: Addison-Wesley Publishing, 1993.

DODGSON, M.; GANN, D; SALTER, A. The management of technological innovation: strategy and practice. 2 ed. New York: Oxford, 2008.

ELKINGTON, J. Cannibals with forks: the triple bottom line of $21^{\text {st }}$ century business. Capstone Publishing: Oxford, 1997.

FLICK, U. Uma introdução à pesquisa qualitativa. 2. ed. Porto Alegre: Bookman, 2004.

FUSSLER, C.; JAMES, P. Driving eco innovation: a breakthrough discipline for innovation and sustainability. London: Pitman Publishing, 1996. 
GOBBLE, M. M. Innovation and sustainability. Research Technology Management, USA, v. 55, n.5, p. 64- 66, Sept./Oct., 2012.

HALL, J.; VRENDENBURG, H. The challenges of innovations for sustainable development. Sloan Management Review, Massachusetts, v. 45, n. 1, p. 61-68, Oct. 2003.

HANSEN, E. G.; GROSSE-DUNKER, F.; REICHWALD, R. Sustainability innovation cube: a framework to evaluate sustainabilityoriented innovations. International Journal of Innovation Management, London, v. 13, n. 4, p. 683-713, Dec. 2009.

HART, S. Capitalism at the Crossroads: the unlimited business opportunities in solving the world's most difficult problems. Upper Saddle River, New Jersey: Wharton School Publishing, 2005.

HART, S.; MISLTEIN, M. B. Criando valor sustentável. RAE Executivo, São Paulo v. 3, n. 2, p. 65-79, maio/jul. 2004.

HAUTAMÄKI, A. Sustainable innovation. Helsinki: Prima, 2010.

HELFAT, C. E.; PETERAF, M. A. Understanding dynamic capabilities: progress along a developmental path. Strategic Organization. Thousand Oaks, v. 7, n.1, p. 91-102, Feb. 2009.

HELLSTRÖM, T. Dimensions of environmentally sustainable innovation: the structure of eco-innovation concepts. Sustainable Development, Sydney, v. 15, n. 3, p. 148-159, May/June 2007.

LAWSON, B.; SAMSON, D. Developing innovation capability in organizations: a dynamic capabilities approach. International Journal of Innovation Management, London, v. 5 , n.3, p. 377-400, Sept. 2001.

NIDUMOLU, R.; PRAHALAD, C. K.; RANGASWAMI, M. R. Why sustainability is now the key driver of innovation. Harvard Business Review, Massachusetts, v. 49 n. 4, p. 1-9, Sept. 2009. 
ORGANIZAÇÃO DAS NAÇÕES UNIDAS - ONU. Objetivos de milênio. 2000. Disponível em: <http://www.objetivosdomilenio.org.br/>. Acesso em: 2 jan. 2013.

OPPEN, C. V.; BRUGMAN, L. Organizational capabilities as the key to sustainable innovation. In: ISPIM CONFERENCE - SUSTAINABILITY IN INNOVATION: INNOVATION MANAGEMENT CHALLENGES, 22., 2011, Hamburg-Germany. Anais...Hamburg-Germany : The Hamburg University of Technology, 12-15 June de 2011. Disponível em: <www.ispin.org/members/proocedings/ISPIM2011/commonfiles/ files/320710409_Paper.pdf Acesso em: 02 Mar. 2013.

BRASIL. PROGRAMA DAS NAÇÕES UNIDAS PARA O DESENVOLVIMENTO (PNUD). Os 8 objetivos de desenvolvimento do milênio. 2010. Disponível em: <http://www.pnud.org.br/ODM8. aspx>. Acesso em: 10 Dez. 2013.

PORTER, M. E.; KRAMER, M. R. Strategy e society: the link between competitive advantage and corporate social responsibility. Harvard Business Review, Massachusetts, v. 84, n.12,p. 78-92, Dec. 2006.

PRAHALAD, C. K. A Riqueza na Base da Pirâmide: como erradicar a pobreza com o lucro. Porto Alegre: Bookman, 2005.

SAVITZ, A. W.; WEBER, K. A empresa sustentável: o verdadeiro sucesso é o lucro com responsabilidade social e ambiental. Rio de Janeiro: Elsevier, 2007.

SHAH, S. K.; CORLEY, K. G. Building better theory by bridging the quantitative-qualitative divide. Journal of Management Studies, Massachusetts, v. 43, n.8, p. 1821-1835, Dec. 2006.

SELLTIZ, C.; JAHODA, M.; COOK, S. W. Métodos de pesquisa nas relações sociais. São Paulo: EPU, 1965.

SIMONS, L.; SLOB, A.; HOLSWILDER, H.; TUKKER, A. The fourth generation: new strategies call for new eco-indicators. Environmental 
Quality Management, Massachusetts, v. 11 , n. 2, p. 51-61, Dec. 2001.

SZEKELY, F. Strategic innovation for sustainability: tomorrow's challenges.International Institute for Management Development: Research Associate Heidi Strebel, June 2012. Disponível em: <http:// www.imd.org/research/challenges/strategic-innovation-sustainabilityfrancisco-szekely-heidi-strebel.cfm>.Acesso em: 02 Mar. 2013.

TEECE, D.; PISANO, G., SHUEN, A. Dynamic capabilities and strategic management. Strategic Management Journal, Massachusetts, v. 18, n.7, p. 509-533, Aug.1997.

TEECE, D. J. Explicating dynamic capabilities: The nature and microfoundations of (sustainable) enterprise performance. Strategic Management Journal, Massachusetts, v. 28, n.13, p.1319-1350, Aug. 2007.

TIDD, J.; BESSANT, J.; PAVITT, K. Gestão da Inovação. 3.ed. Porto Alegre: Bookman, 2008.

BRAZIL. UNITED NATIONS CONFERENCE ON SUSTAINABLE DEVELOPMENT - UNCED. Rio 92. 3-4 june 1992. Rio de Janeiro. Disponível em <http://www.un.org/geninfo/bp/enviro.html>. Acesso em: 02 jan. de 2013.

BRAZIL. UNITED NATIONS CONFERENCE ON SUSTAINABLE DEVELOPMENT - UNCED. Rio+20. 20-22 June 2012. Rio de Janeiro. Disponível em <https://sustainabledevelopment.un.org/rio20.html>. Acesso em: 02 jan. de 2013.

Artigo recebido em: 14/09/2015

Aprovado em: 18/12/2015 